\title{
The importance of standardized interpretation of molecular breast imaging with dedicated gamma cameras
}

\author{
Orazio Schillaci
}

Published online: 14 March 2012

(C) Springer-Verlag 2012

Breast cancer (BC) is the most common malignancy and the second leading cause of cancer death in females, accounting in the USA for $29 \%$ of the total new cancer cases and $14 \%$ of the total cancer deaths in 2012 [1]. Early diagnosis and treatment are of the utmost importance to improve prognosis.

For detection and characterization of primary breast lesions, anatomical imaging including mammography, ultrasonography, and MRI are commonly employed. Scintimammography is a molecular nuclear medicine technique for breast imaging which uses single-photon radiopharmaceuticals such as ${ }^{99 \mathrm{~m}} \mathrm{Tc}$ sestamibi and ${ }^{99 \mathrm{~m}} \mathrm{Tc}$ tetrofosmin. It is a functional imaging modality so some of the main drawbacks of mammography are resolved [2]. It was developed almost 20 years ago with standard large field-of-view (FOV) gamma cameras. Certainly, the principal limiting factor in the clinical acceptance of scintimammography has been its low sensitivity for cancers of $\leq 1 \mathrm{~cm}$ in size, mainly because of the lack of suitable equipment specifically designed for breast imaging.

Dedicated gamma cameras specifically built for breast imaging are now available. The use of these breast-optimized, small FOV, high-resolution cameras allows both greater flexibility in patient positioning (improving breast imaging by limiting the FOV and reducing image contamination from other organs, i.e. liver and heart) and breast compression, with

\section{O. Schillaci $(\square)$}

Department of Biopathology and Diagnostic Imaging,

University "Tor Vergata",

Viale Mazzini 121,

00195 Rome, Italy

e-mail: orazio.schillaci@uniroma2.it

O. Schillaci

IRCCS Neuromed,

Pozzilli, Italy an important increase in the target-to-background ratio [3]. In fact, the detector can be placed directly against the chest and a mild compression is possible, to reduce breast thickness and improve the camera's sensitivity. Moreover, by design, these specific cameras are also able to provide better intrinsic and extrinsic spatial resolution than standard ones, with an enhancement in contrast resolution for small lesions [4], and to acquire projections similar to those of mammography (craniocaudal, mediolateral oblique and true lateral).

Studies comparing standard and breast-specific cameras have provided clinical evidence that the dedicated ones show better accuracy in molecular breast imaging, especially in increasing the sensitivity for subcentimetre lesions [4-6]. It is worth noting that a recent retrospective review of one institution's experience with breast-specific gamma imaging (BSGI) has indicated that this test is not only both sensitive (93\%) and specific (79\%) for the identification of BC, but it is also helpful as an adjunct to standard breast imaging modalities for problem solving in indeterminate cases [7]. Moreover, in this study including 416 cases, BSGI was demonstrated to be useful in evaluating lesions difficult to biopsy and in patients who desired further testing rather than biopsy or short term follow-up of their breast abnormality.

These interesting data have been then confirmed in a multicentre clinical patient registry analysis, which aimed to quantify the impact of BSGI on the management of patients with $\mathrm{BC}$ in clinical practice, and to identify the subgroups benefiting more from the use of this examination [8]. A total of 1,042 patients were evaluated. All of them had at least one (the majority two or more) of the following indications: equivocal mammography or ultrasonography findings; a personal history of $\mathrm{BC}$; a family history or other factors establishing a high risk of developing $\mathrm{BC}$; a recent positive mammogram; clinical findings such as a palpable mass, breast pain or bloody nipple discharge; dense breast 
tissue difficult to image by mammography. BSGI was positive in 408 patients (227 malignant or high-risk lesions requiring additional intervention), negative in 634 patients (23 with malignant or high-risk lesions), and indeterminate in 69 patients (all benign lesions), with an overall sensitivity of $91 \%$ and a specificity of $77 \%$. In particular, BSGI significantly contributed to the detection of malignant and high-risk lesions in patients with a negative or indeterminate mammogram, and it improved management in patients with indeterminate mammographic findings when compared with ultrasonography.

Although BSGI is still not widely available or routinely used, in 2010 the Society of Nuclear Medicine (SNM) published a practice guideline for BSGI to assist breast imaging practitioners in selecting patients, and for performing, interpreting and reporting this examination [9]. In the SNM guideline, interpretation criteria for BSGI are included, suggesting the importance of having a common language for the description of findings. This issue is clearly highlighted in the paper by Conners et al. [10] published in the current issue of the European Journal of Nuclear Medicine and Molecular Imaging, which proposes a lexicon for standardized interpretation of BSGI, selecting cases from a database of more than 3,000 examinations.

The need for consistent and universally understood terminology in breast imaging was recognized by the American College of Radiology (ACR), which, in an attempt to achieve uniformity in mammographic interpretation, proposed the Breast Imaging Reporting and Data System (BI-RADS) to describe the radiologist's level of suspicion about the mammographic findings [11]. This initiative was the first practice management system developed for imaging, and it was begun to address the lack of standardization in mammography practice reporting. An important component of the BI-RADS is the lexicon, a dictionary of descriptors of specific imaging features, but it also contains a suggested reporting structure (with final assessment categories and accompanying management recommendations), and a framework for data collection and auditing. The establishment of this lexicon has provided new opportunities for communication, quality assurance, research and improved patient care.

After the initial creation of BI-RADS for mammography in 1992 [11], three more editions were published in 1995, 1998 and 2003 [12]. Subsequently, the need for a breast ultrasonography lexicon was recognized by the ACR, and using techniques similar to those for the mammography BIRADS, agreement on terminology and assessment categorization was reached by consensus of an expert working group and its subcommittee on lexicon development. As a result of similar problems in reporting breast abnormalities on MRI, a lexicon for contrast-enhanced breast MRI was also developed. The objective of the ACR BI-RADS lexicon for MRI is to standardize the language in breast MRI reporting, to aid clinicians in understanding the results of breast
MRI for subsequent patient management, and in scientific research to aid comparison of studies using similar terminology [12].

The BI-RADS assessment has been shown to be very useful for improving the quality of breast image interpretation (mammography, ultrasonography and MRI) by providing a standard language which can be adopted to compare findings across multiple scientific studies and enable all radiologists to describe imaging findings in a consistent manner. Moreover, the BI-RADS final assessment categories and their accompanying management recommendations have become the standard by which physicians determine breast care on the basis of imaging [12]. Therefore, the implementation of a lexicon for describing BSGI as proposed by Conners et al. [10] is highly advisable for the development of this technique, because it is necessary that the terminology is easy to understand and consistently used by interpreting nuclear medicine physicians. On the other hand, a similar lexicon has recently been published for the standardized reporting of the findings of positron emission mammography (PEM), an emerging molecular imaging technology which produces high-resolution tomographic images of ${ }^{18} \mathrm{~F}-\mathrm{FDG}$ uptake in the breasts [13].

With increasing use of BSGI, there is clearly a need for standardized terminology to describe its outcomes, for interpretation and to provide management recommendations, similar to the standardized classification that already exists for other breast imaging modalities. The lexicon of Conners et al. [10] includes descriptors of lesion type, location, size, features and radiopharmaceutical uptake both in lesions and background parenchyma, and associated findings, and it was developed by three fellowship-trained, dedicated breast radiologists (blinded for review). It was constructed taking into account the terms adopted for the other BI-RADSs and with the aim of being unified with the lexicon for PEM [13].

Variability in BSGI interpretation can be due both to differences in detection of lesions and to variations in lesion characterization and subsequent management. The descriptors in the lexicon should be selected on the basis of their ability to discriminate between benign and malignant findings, in order to achieve a high sensitivity (true-positive rate) that is not at the expense of a low specificity (falsepositive rate). In the study by Conners et al. [10], for six independent observers, the median sensitivity was $100 \%$, the specificity was $88 \%$, the negative predictive value was $100 \%$ and the positive predictive value was $85 \%$. The six breast imaging radiologist observers did not have any prior experience in interpreting BSGI and had only attended a 2$\mathrm{h}$ didactic BSGI training session. These findings are clearly of value, and indicate that dedicated breast imaging radiologists, newly trained to evaluate BSGI using the proposed lexicon, are able to obtain a high diagnostic accuracy.

However, it is important for wider clinical acceptance of the standardized interpretation, that a similar study should 
be performed in a larger series of patients, in particular including a high number of $\mathrm{BCs}<1 \mathrm{~cm}$ in size. In their patient population, Conners et al. [10] evaluated $\mathrm{BCs}$ ranging in size from 1 to $6.3 \mathrm{~cm}$, but BSGI also has the potential to yield an improved sensitivity in the detection of subcentimetre malignant lesions. In a group of 124 patients with BC, BSGI was able to detect 41 out of $45(91.1 \%)$ malignant lesions $\leq 1 \mathrm{~cm}$ and $98 / 98(100 \%)$ malignant lesions $>1 \mathrm{~cm}$, and in relation to palpability, the examination identified 38 out of 41 (92.7\%) nonpalpable lesions and 101 out of 102 (99\%) palpable lesions [14]. Moreover, it will surely also be interesting to evaluate and validate the use of this new lexicon among nuclear medicine physicians with previous experience in standard scintimammography and/or in BSGI but not dedicated only to breast imaging.

Another fundamental outcome of the study by Conners et al. [10] is the interobserver agreement for final assessment of BSGI alone, i.e. without mammography, with a mean kappa of 0.8, and the agreement of observers with expert consensus (kappa 0.83). This interobserver agreement is higher than that reported in validation studies of other breast imaging modalities [10], including PEM [15], and also when compared to a previous BSGI report evaluating BC screening in women with mammographically dense breasts [16]. In this latter study, two observers retrospectively examined images obtained with a dedicated dual-head gamma camera for the presence of abnormal radiopharmaceutical uptake, using a five-point score $(1=$ no abnormality, $5=$ highly suspicious for malignancy). Nevertheless, the patient population evaluated in this screening study was completely different from that included in the study by Conners et al. (11 BCs in 936 women vs. 20 malignant lesions in 50 breasts, respectively). Finally, it is also of the utmost importance to validate the use of the new lexicon among observers from different institutions.

When we look at the results of lexicon agreement in specific fields, there was a substantial interobserver agreement for lesion type, distribution of non-mass lesions and lesion intensity, but it was only fair for background parenchymal uptake (kappa 0.31). The limits of the description of the degree of radiopharmaceutical uptake in the normal breast parenchyma, with the subcutaneous fat as reference, could be overcome by the quantification of uptake. Recently, the development of a dual-head camera for BSGI has allowed the simultaneous acquisition of opposing breast views, providing the quantification of lesion parameters including size, depth to the collimator face and relative tracer uptake [17]. Validation of the methods by Monte Carlo and phantom simulations have shown that using the measured lesion diameter and measurements of counts in the lesion and background breast region, relative radiotracer uptake and tumour to background ratio can be accurately calculated [18].
In the final assessment of BSGI interpreted with correlating mammograms the agreement was almost perfect (kappa 0.87). The fact that only four cases were given an assessment of 3 (i.e. probably benign) when BSGI was interpreted in conjunction with mammography clearly indicates that nuclear breast imaging techniques should be performed only as an adjunct to mammography in specific clinical indications, and not as a first-line test to detect $\mathrm{BC}$, and that the integration of functional and anatomic images is also able to improve the diagnostic accuracy of breast imaging.

Additionally, the introduction of the subcategories $4 \mathrm{a}, 4 \mathrm{~b}$ and $4 \mathrm{c}$ has been beneficial in stratifying the likelihood of malignancy in lesions recommended for biopsy. In fact, the lesions of category 4 are suspicious abnormalities which do not have the characteristic morphologies of $\mathrm{BC}$, but have a definite low to moderate probability of being malignant. BSGI may result in sufficient concern for the breast imaging practitioner to urge a biopsy, and if possible the relevant probabilities should be cited so that the patient and her physician can make the decision on the ultimate course of action. The agreement among observers for the presence of associated findings should be further evaluated, especially regarding the uptake in the axilla, which can be related to the presence of lymph-node metastases. The possible clinical role of BSGI in detecting such metastases deserves more studies [19].

Because interobserver agreement with the proposed lexicon is good, its use may provide accurate and consistent descriptions and assessment for BSGI. All other systems which were previously used often contain subjective and undefined terms. This new lexicon provides a standardized language for the interpretation of BSGI, so reducing confusion, and it is a quality assurance tool also designed to standardize reporting and facilitate outcome monitoring. Nevertheless, only the development and large availability of BSGI will allow this modality to really become routine in breast imaging. Moreover, to be clinically important, BSGI will need to show equivalent performance at decreased radiation doses [20]. Therefore, multicentre studies in larger series should be performed before BSGI can be definitively proposed as a diagnostic modality for $\mathrm{BC}$ detection, particularly at an early stage, taking into account the possible future role of PEM in this clinical scenario.

In conclusion, the addition of the lexicon proposed by Conners et al. for BSGI [10] is helpful and could be used with good agreement among nuclear medicine physicians, even those without previous specific training in this new terminology, after validation in this specific group. In the near future, it will be important to determine whether training in this lexicon's feature analysis would improve observer agreement with experienced breast imagers in BSGI lesion description and final assessments (management recommendations). It is worth noting that even experienced breast imagers show 
improved agreement in feature analysis and final assessment following BI-RADS training [21]. Moreover, continued development of methods to improve standardization of BSGI interpretation is needed. However, the lexicon should be arranged so as to be adopted in everyday practice, and its constant use should make it possible to issue meaningful, unambiguous breast BSGI reports. The dissemination of knowledge of the BSGI lexicon could also facilitate the creation of databases for longitudinal studies relating imaging findings with pathological outcomes. Once established, the lexicon will facilitates the comprehension of BSGI results by all members of the multidisciplinary breast care team (radiologists, surgeons, pathologists, oncologists, radiation oncologists and others), so improving patient care.

\section{References}

1. Siegel R, Naishadham D, Jemal A. Cancer statistics, 2012. CA Cancer J Clin. 2012;62:10-29.

2. Schillaci O, Buscombe JR. Breast scintigraphy today: indications and limitations. Eur J Nucl Med Mol Imaging. 2004;31 Suppl 1: S35-45.

3. Schillaci O, Cossu E, Buonomo O, Granai AV, Pistolese CA, Danieli R, et al. Dedicated breast camera: is it the best option for scintimammography? J Nucl Med. 2005;46:550.

4. Brem RF, Schoonjans JM, Kieper DA, Majewski S, Goodman S, Civelek C. High-resolution scintimammography: a pilot study. J Nucl Med. 2002;43:909-15.

5. Schillaci O, Cossu E, Romano P, Sansò C, Danieli R, Granai AV, et al. High-resolution gamma-camera for molecular breast imaging: first clinical results. Phys Med. 2006;21 Suppl 1:121-4.

6. Spanu A, Cottu P, Manca A, Chessa F, Sanna D, Madeddu G. Scintimammography with dedicated breast camera in unifocal and multifocal/multicentric primary breast cancer detection: a comparative study with SPECT. Int J Oncol. 2007;31:369-77.

7. Siegal E, Angelakis E, Morris P, Pinkus E. Breast molecular imaging: a retrospective review of one institutions experience with this modality and analysis of its potential role in breast imaging decision making. Breast J. 2012. doi: 10.1111/j.1524-4741.2011.01214.x

8. Weigert JM, Bertrand ML, Lanzkowsky L, Stern LH, Kieper DA. Results of a multicenter patient registry to determine the clinical impact of breast-specific gamma imaging, a molecular breast imaging technique. AJR Am J Roentgenol. 2012;198:W69-75.
9. Goldsmith SJ, Parsons W, Guiberteau MJ, Stern LH, Lanzkowsky $\mathrm{L}$, Weigert $\mathrm{J}$, et al. SN practice guideline for breast scintigraphy with breast-specific gamma-cameras. J Nucl Med Technol. 2010;38:219-24.

10. Conners AL, Hruska CB, Tortorelli CL, Maxwell RW, Rhodes DJ, Boughey JC, et al. Lexicon for standardized interpretation of gamma camera molecular breast imaging: observer agreement and diagnostic accuracy. Eur J Nucl Med Mol Imaging. 2012. doi: 10.1007/s00259-011-2054-Z

11. American College of Radiology. Breast Imaging Reporting and Data System (BI-RADS). Reston, Va: American College of Radiology; 1992.

12. Burnside ES, Sickles EA, Bassett LW, Rubin DL, Lee CH, Ikeda DM, et al. The ACR BI-RADS experience: learning from history. J Am Coll Radiol. 2009;6:851-60.

13. Narayanan D, Madsen KS, Kalinyak JE, Berg WA. Interpretation of positron emission mammography: feature analysis and rates of malignancy. AJR Am J Roentgenol. 2011;196:956-70.

14. Spanu A, Chessa F, Meloni GB, Sanna D, Cottu P, Manca A, et al. The role of planar scintimammography with high-resolution dedicated breast camera in the diagnosis of primary breast cancer. Clin Nucl Med. 2008;33:739-42.

15. Narayanan D, Madsen KS, Kalinyak JE, Berg WA. Interpretation of positron emission mammography and MRI by experienced breast imaging radiologists: performance and observer reproducibility. AJR Am J Roentgenol. 2011;196:971-81.

16. Rhodes DJ, Hruska CB, Phillips SW, Whaley DH, O'Connor MK. Dedicated dual-head gamma imaging for breast cancer screening in women with mammographically dense breasts. Radiology. 2011;258:106-18.

17. Hruska CB, Phillips SW, Whaley DH, Rhodes DJ, O'Connor MK. Molecular breast imaging: use of a dual-head dedicated gamma camera to detect small breast tumors. AJR Am J Roentgenol. 2008;191:1805-15.

18. Hruska CB, O'Connor MK. Quantification of lesion size, depth, and uptake using a dual-head molecular breast imaging system. Med Phys. 2008;35:1365-76.

19. Spanu A, Chessa F, Sanna D, Cottu P, Manca A, Nuvoli S, et al. Breast cancer axillary lymph node metastasis detection by a highresolution dedicated breast camera: a comparative study with SPECT and pinhole SPECT. Cancer Biother Radiopharm. 2007;22:799-811.

20. Hendrick RE. Radiation doses and risks from breast imaging studies. Radiology. 2010;257:246-53.

21. Berg WA, D’Orsi CJ, Jackson VP, Bassett LW, Beam CA, Lewis $\mathrm{RS}$, et al. Does training in the Breast Imaging Reporting and Data System (BI-RADS) improve biopsy recommendations or feature analysis agreement with experienced breast imagers at mammography? Radiology. 2002;224:871-80. 